\title{
4-methylumbelliferone inhibits clonogenic potency by suppressing high molecular weight-hyaluronan in fibrosarcoma cells
}

\author{
KAZUKI HASEGAWA $^{1}$, RYO SAGA ${ }^{1}$, REI TAKAHASHI ${ }^{1}$, ROMAN FUKUI ${ }^{1}$, \\ MITSURU CHIBA $^{2}$, KAZUHIKO OKUMURA ${ }^{3}$, EICHI TSURUGA ${ }^{1}$ and YOICHIRO HOSOKAWA ${ }^{1}$
}

\begin{abstract}
Departments of ${ }^{1}$ Radiation Sciences, and ${ }^{2}$ Bioscience and Laboratory Medicine, Graduate School of Health Sciences, Hirosaki University, Hirosaki, Aomori 036-8564; ${ }^{3}$ Department of Oral and Maxillofacial Surgery, School of Dentistry, Health Science University of Hokkaido, Tobetsu, Hokkaido 061-0293, Japan
\end{abstract}

Received August 23, 2019; Accepted January 21, 2020

DOI: $10.3892 / \mathrm{ol} .2020 .11370$

\begin{abstract}
The inflammatory response is closely associated with cancer cell survival. It has been reported that inflammatory signaling cascades promote tumor survival and exert detrimental effects in normal tissue. Hyaluronans have different cellular functions depending on their molecular weights and high molecular weight-hyaluronan (HMW-HA) exhibits anti-inflammatory effects. A previous study determined that the co-administration of 4-methylumbelliferone (4-MU) and X-ray irradiation enhanced anti-tumor and anti-inflammatory effects in HT1080 human fibrosarcoma cells. However, many mechanisms underlie the effect of hyaluronan molecular weight on cells and the induction of anti-inflammatory effects via 4-MU. The present study aimed to determine the relationship between hyaluronan synthesis inhibition by 4-MU and its anti-inflammatory and radio-sensitizing effect in the context of hyaluronan molecular weight. The hyaluronan concentration following 2 Gy X-ray irradiation and/or 4-MU administration was analyzed via ELISA. Additionally, the mRNA expressions of hyaluronan synthase (HAS) by 4-MU and various inflammatory cytokines and interleukins (IL) following exogenous HMW-HA administration were evaluated via Reverse transcription-quantitative PCR. Invasive potential was assessed by matrigel transwell assays and cell survival following exposure to 4-MU with HMW-HA was determined using a clonogenic potency assay. The results of the present study demonstrated that
\end{abstract}

Correspondence to: Dr Ryo Saga or Professor Yoichiro Hosokawa, Department of Radiation Sciences, Graduate School of Health Sciences, Hirosaki University, 66-1 Hon-cho, Hirosaki, Aomori 036-8564, Japan E-mail: sagar@hirosaki-u.ac.jp

E-mail: hosokawa@hirosaki-u.ac.jp

Key words: inflammatory cytokine, 4-methylumbelliferone, hyaluronan, interleukin
4-MU suppressed HMW-HA production by inhibiting HAS2 and HAS3 expression. In addition, the surviving fraction of fibrosarcoma cells were rescued from the cell-killing effect of 4-MU via the exogenous administration of HMW-HA. The mRNA levels of certain inflammatory cytokines, including IL-1 $\alpha$, IL-36 $\gamma$ and IL-37 were elevated following HMW-HA administration. The surviving fraction of cells irradiated with 2 Gy alone did not increase following exogenous HMW-HA administration. The results of the present study indicated that the radio-sensitizing effect of 4-MU and the inhibitory effect on hyaluronan synthesis were not closely associated. It was also revealed that IL-1 $\alpha$, IL-36 $\gamma$ and IL-37 were associated with the cell-killing effect of 4-MU in HT1080 cells.

\section{Introduction}

In recent years, high-precision radiotherapy has played an important role in cancer treatment. However, recurrence and distant metastasis caused by residual cancer cells remain major concerns and lead to a poor outcome (1). It has been reported that inflammatory signaling cascades promote tumor survival, while inducing detrimental effects in normal tissues (2). Further, inflammatory response has been reported to play a crucial role in different stages of tumor development, including, initiation, transformation, invasion, and metastasis, and is known to promote angiogenesis, proliferation, and resistance to apoptosis $(3,4)$. These factors contribute to poor prognosis post radiotherapy. Therefore, targeting the inflammatory signaling pathway could inhibit angiogenesis, proliferation, and resistance to apoptosis and help radio-sensitize the tumor cells. In addition, this targeting could preferentially radio-sensitize the tumor cells without affecting the normal cells.

4-Methylumbelliferone (4-MU) is a hyaluronan (HA) synthesis inhibitor that has been demonstrated to possess anti-tumor and anti-invasion/metastatic effects by blocking the interaction between hyaluronan and CD44 and suppressing downstream signaling (5). In addition, 4-MU has been reported to inhibit HA synthesis (HAS) (6), and especially HAS2 that is involved in synthesis of high molecular 
weight-HA (HMW-HA) $(7,8)$. These references reported that the interaction between hyaluronan and its receptor CD44 in bone metastasis and the characteristics of the HAS including HAS1, HAS2, and HAS3. HA has unique biological effects on cells, depending on the molecular weight. Generally, it has been reported that low molecular weight-HA (LMW-HA) has pro-inflammatory, pro-angiogenic, and pro-tumorigenic effects, while HMW-HA has anti-inflammatory and anti-tumor effects (9). However, many researchers have identified that these effects are not entirely accurate. A recent study demonstrated that HMW-HA correlates with poor survival in patients with pancreatic cancer (10). In addition, it has been shown that 4-MU suppresses inflammatory cytokines such as IL-6, -8 and chemokines, and has anti-inflammatory effects (11). The anti-inflammatory effects of 4-MU may be associated with its ability to block the synthesis of endogenous HA and inhibit the lipopolysaccharide (LPS)-induced up-regulation of inflammatory mediators and inflammatory-related receptors, like the toll-like receptor 4 (TLR4). It has been reported that nuclear factor kappa-light-chain (NF- $\varkappa \mathrm{B})$, a transcription factor, is activated via the IL-6 signaling network and promotes the formation of cancer stem cells and mesenchymal stem cells (12). Moreover, IL-6 activates the phosphorylation of signaling pathways associated with cancer survival, such as PI3K/Akt and JAK/STAT, upon binding IL-6R. In addition, overexpression of IL-6 induced higher distant metastasis by cancer cells $(13,14)$.

In our previous study, administration of a combination of $100 \mu \mathrm{M} 4-\mathrm{MU}$ and $2 \mathrm{~Gy} \mathrm{X}$-ray irradiation caused downregulation of matrix metalloproteinases-2 and -9 and inhibited the colony-forming and metastatic potential of HT1080 human fibrosarcoma cells (15). Although many studies reveal the relationship cancer mechanism and hyaluronan, there are few studies that the effect of HA synthesis inhibition and irradiation. In addition, the relationship between 4-MU mediated inhibition of hyaluronan synthesis and the consequent inflammatory and radio-sensitizing effects remains unclear. Therefore, we investigated the sensitization mechanism of 4-MU in HT1080 cells and sought to clarify these relationships in this study.

\section{Materials and methods}

Reagents. 4-MU was purchased from Nacalai Tesque, and diluted in dimethylsulfoxide (DMSO) (Wako Pure Chemical Industries, Ltd.) at a working concentration of $500 \mu \mathrm{M}$. We used DMSO at a final concentration of $0.05 \%$. To clearly observe the effects of4-MU, $500 \mu \mathrm{M}$ 4-MU was used, which also minimized the cytotoxic effects on normal fibroblast cells (16). LMW-HA (15-40 kDa, cat. no. GLR001) and HMW-HA ( $>950 \mathrm{kDa}$, cat. no. GLR002) were purchased from R\&D Systems and used at working concentrations of $200 \mu \mathrm{g} / \mathrm{ml}$ and $100 \mu \mathrm{g} / \mathrm{ml}$, respectively.

Cell culture. HT1080 human fibrosarcoma cells from American Type Culture Collection were cultured in Roswell Park Memorial Institute (RPMI) 1640 medium (Thermo Fisher Scientific Inc.) supplemented with $10 \%$ heat-inactivated fetal bovine serum (FBS; Japan Bio Serum) and $1 \%$ penicillin/streptomycin (Life Technologies) at $37^{\circ} \mathrm{C}$ in a humidified atmosphere of $5 \% \mathrm{CO}_{2}$. HT1080 cell is suitable for verifying the hyaluronan synthesis inhibition effect of 4-MU because it has a high self-hyaluronan production and high invasion abilities.

Clonogenic potency assay. The clonogenic potency of HT1080 cells was evaluated using the colony formation assay. We seeded the control group with 300 cells, the $500 \mu \mathrm{M}$ 4-MU group with 20000 cells, the 2 Gy X-ray irradiation group with 600 cells, and the 4-MU and 2 Gy X-ray irradiation group with 50000 cells on $\phi 60$ culture dishes and incubated for $2 \mathrm{~h}$, and then subjected to X-ray irradiation at $2 \mathrm{~Gy}$ in the presence of $500 \mu \mathrm{M} 4-\mathrm{MU}$ and $200 \mu \mathrm{g} / \mathrm{ml}$ LMW-HA or $100 \mu \mathrm{g} / \mathrm{ml}$ HMW-HA, followed by further incubation for $24 \mathrm{~h}$. The 4-MU was then washed out. To form the appropriate number of colonies, HT1080 cells was seeded different numbers because of cell killing effects of 4-MU. In addition, we determined the exogenous HA concentration and use it according to the procedure reported previously $(5,17)$. We tried to use HMW-HA at the same concentration as LMW-HA, but it did not dissolve at a concentration of $200 \mu \mathrm{g} / \mathrm{ml}$. Thus, we used $200 \mu \mathrm{g} / \mathrm{ml}$ LMW-HA or $100 \mu \mathrm{g} / \mathrm{ml}$ HMW-HA. After 7-10 days of incubation, cells were fixed with methanol (Wako Pure Chemical Industries Ltd.), stained with Giemsa (Wako Pure Chemical Industries Ltd.). Colonies consisting of $>50$ cells were counted. The survival fraction for HT1080 cells was calculated as the plating efficiency of the irradiated and/or 4-MU administration samples compared with that of the control samples.

Irradiation. Ionizing radiation (IR) was delivered using an X-ray generator (MBR-1520R-3; Hitachi Medical Co.) with 0.5-mm aluminum and $0.3-\mathrm{mm}$ copper filters at a distance of $45 \mathrm{~cm}$ between the focus and target $(150 \mathrm{kV}, 20 \mathrm{~mA}, 1.0 \mathrm{~Gy} / \mathrm{min})$. During X-ray exposure, the total dose and dose rate were monitored with a thimble ionization chamber (MZ-BD-3; Hitachi Medical Co.) placed next to the sample. We use the dose rate in air, where air kerma is converted to dose rate.

HA density quantitation. HA concentration was measured by using the Hyaluronan Quantikine ${ }^{\circledR}$ ELISA kit (R\&D Systems, Inc.) using the procedure reported previously (15). HA concentration was calculated from a standard curve of the measured at $450 \mathrm{~nm}$ absorbance.

RNA extraction and analysis. Total RNA was extracted from HT1080 cells, and RNA quality was confirmed according to the procedure reported previously (15).

Cyanine (Cy)3-labeled cRNA samples were synthesized from total RNA, and hybridized to $8 \times 60 \mathrm{~K}$ format SurePrint G3 Human GE v2 microarray slides (eArray Design $\mathrm{ID}=039494$ ) according to the manufacturer's instructions (Agilent Technologies Inc.). Cy3 fluorescence was detected with a DNA microarray scanner (G2600A SureScan), and processed using Feature Extraction software (both from Agilent Technologies Inc.).

Reverse transcription-quantitative polymerase chain reaction (RT-qPCR). cDNA was synthesized using a procedure reported previously with reaction mixture containing forward and reverse primers (Table I) (15). The reaction was carried out 
Table I. Primer sequence of the target gene.

Primers

Sequence (5'-3')

HAS 1 forward
HAS 1 reverse
HAS 2 forward
HAS 2 reverse
HAS3 forward
HAS3 reverse
IL-1 $\alpha$ forward
IL-1 $\alpha$ reverse
IL-1 $\beta$ forward
IL-1 $\beta$ reverse
IL-6 forward
IL-6 reverse
IL-36 $\alpha$ forward
Il-36 $\alpha$ reverse
IL-36 $\gamma$ forward
IL-36 $\gamma$ reverse
IL-37 forward
IL-37 reverse
GAPDH forward
GAPDH reverse

TGTGTATCCTGCATCAGCGGT
CTGGAGGTGTACTTGGTAGCATAACC
CTCCGGGACCACACAGAC
TCAGGATACATAGAAACCTCTCA
ACCATCGAGATGATTCGAGT
CCATGAGTCGTACTTGTTGAGG
GGTTGAGTTTAAGCCAATCCA
TGCTGACCTAGGCTTGATGA
TACCTGTCCTGCGTGTTGAA
TCTTTGGGTAATTTTTGGGATCT
CACTGGGCACAGAACTTATGTTG

AAAATAATTAAAATAGTGTCCTAACGCCAT CATCTACCTGGGCCTGAATG GGGTTGGTTTACAAATCCATTA AAGTGACAGTGTGACCCCAGT GGATTCTGGATTCCCAAATAAA CCCCAGTGCTGCTTAGAAGA

TCACCTTTGGACTTGTGTGAA GTGAGGTCGGAGTCAACG TGAGGTCAATGAAGGGGTC

HAS, hyaluronan synthase. IL, interleukin.

in a real-time PCR system (StepOne Plus; Life Technologies) under the following conditions: $95^{\circ} \mathrm{C}$ for $30 \mathrm{sec}$, followed by 40 cycles of $95^{\circ} \mathrm{C}$ for $5 \mathrm{sec}$, and $54^{\circ} \mathrm{C}$ for $30 \mathrm{sec}$. Target gene expression levels were calculated relative to that of glyceraldehyde 3-phosphate dehydrogenase (GAPDH, internal control) mRNA using the comparative $\Delta \Delta \mathrm{Cq}$ method (18).

Invasion assay. The invasive potential of HT1080 cells was assessed using a BioCoat Matrigel invasion chamber (BD Biosciences). The suspension containing $2.5 \times 10^{4} \mathrm{HT} 1080$ cells was plated in 24-well chambers dishes, and incubated for $22 \mathrm{~h}$ at $37^{\circ} \mathrm{C}$. Invasive cells were fixed and stained using a DiffQuik staining kit (Dade Behring, Inc., Siemens Healthcare $\mathrm{GmbH})$. Invasive cells were counted, and invasion rate was calculated using the procedure reported previously (15).

Statistical analysis. Statistical analysis of microarray data was performed using GeneSpring (Agilent Technologies Inc.). Upand downregulated mRNAs transcripts were selected based on fold change ( $>2$-fold) in irradiated and/or 4-MU-administered samples relative to control samples. Ingenuity Pathway Analysis (IPA; Qiagen Silicon Valley) was used for functional analysis of each transcript. The significance of differences between control and experimental cultures was evaluated using one-way analysis of variance and the Tukey-Kramer test. Statistical analyses were performed using Microsoft Excel 2010 (Microsoft Corporation) with the add-on software Statcel v3 (OMS Publishing). $\mathrm{P}<0.05$ was considered to indicate a statistically significant difference.

\section{Results}

4-MU inhibits HA synthesis and invasion/ metastasis. The HA concentration in the cell culture supernatant following administration of 2 Gy X-ray irradiation and/or 4-MU was analyzed by ELISA. The concentration of HA was significantly decreased in the presence of 4-MU as compared to the control $(22.17 \pm 2.36$ vs. $50.65 \pm 3.90 \mathrm{ng} / \mathrm{ml})$. The concentration of HA in the combination of $500 \mu \mathrm{M} 4-\mathrm{MU}$ and 2 Gy $\mathrm{X}$-ray irradiation treatment was also significantly decreased as compared to 2 Gy X-ray irradiation alone (24.06 \pm 3.94 vs. $54.15 \pm 2.95 \mathrm{ng} / \mathrm{ml}$; Fig. $1 \mathrm{~A})$.

To confirm the effects of 4-MU on HAS, the mRNA expression of HAS1-3 was evaluated by RT-qPCR. The mRNA expression of HAS1 was undetectable (data not shown). The mRNA expression of HAS2 and HAS3 was decreased 0.57 and 0.35 folds, respectively, against the baseline of 4-MU treatment alone (Fig. 1B and C). Furthermore, the expression of HAS2 following treatment with 4-MU and 2 Gy X-ray irradiation was significantly decreased as compared to 2 Gy irradiation alone $(0.27 \pm 0.13$ vs. $1.28 \pm 0.31$; Fig. $1 \mathrm{~B})$. These results suggested that HAS2 and HAS3 expression were significantly decreased by 4-MU.

To verify invasion at the cellular level, the invasion rate potential was measured using a BioCoat Matrigel invasion chamber (Fig. 1D and E). The invasion rate of cells treated with 4-MU was significantly lower than that of the control $(10.8 \pm 2.91$ vs. $30.3 \pm 4.23$ cells in the field of view) (Fig. 1E). In addition, the invasion rate of cells treated 

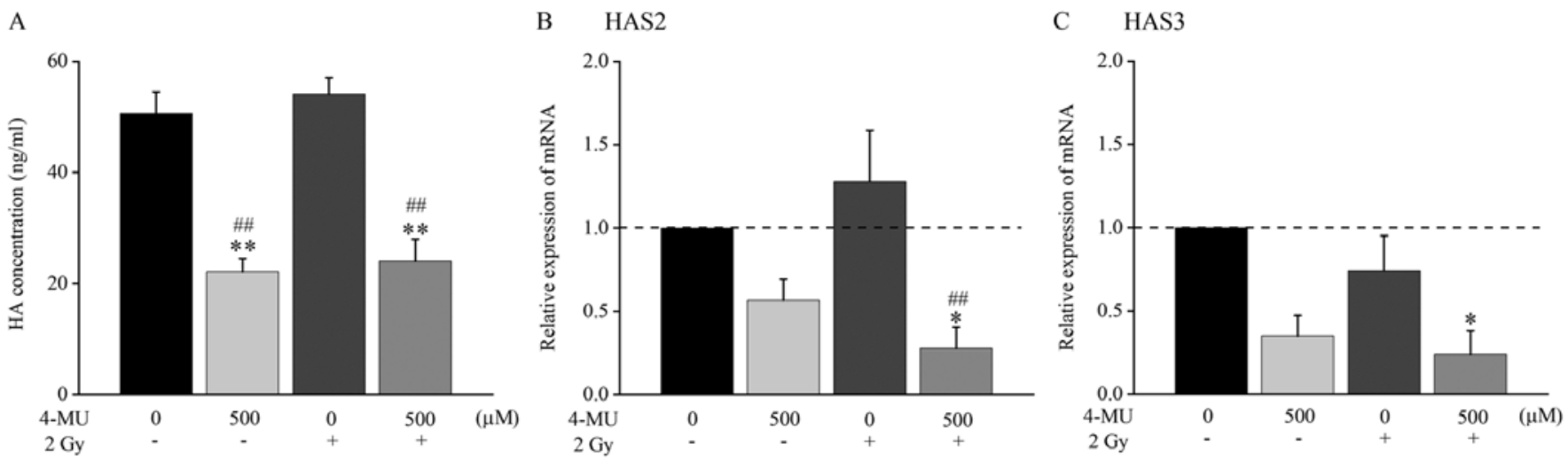

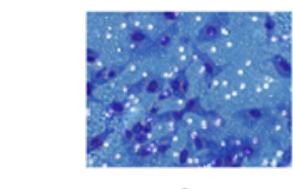

4-MU

0

2 Gy

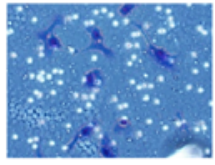

500

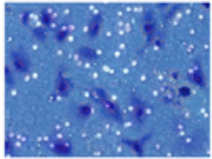

0

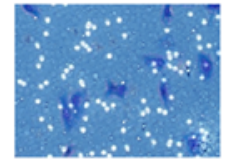

500

$(\mu \mathrm{M})$

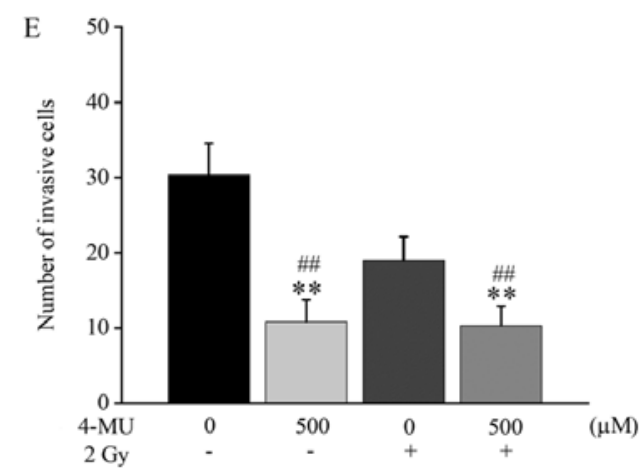

Figure 1. Evaluation of 4-MU effects. (A) Measurement of HA concentration via ELISA. Evaluation of (B) HAS2 and (C) HAS3 mRNA levels, as determined via reverse transcription-quantitative PCR. (D) Representative images of cell invasion observed in the invasion Matrigel chamber, where white dots represent pores and violet points represent the stained invaded cells (magnification, x100). (E) The number of invaded cells was measured. Data are presented as the mean \pm standard deviation. $\mathrm{P}<0.05$ and ${ }^{* *} \mathrm{P}<0.01$ vs. control; ${ }^{\# \#} \mathrm{P}<0.01$ vs. 2 Gy alone. 4-MU, 4-methylumbelliferone; HA, hyaluronan; HAS, hyaluronan synthase.

A

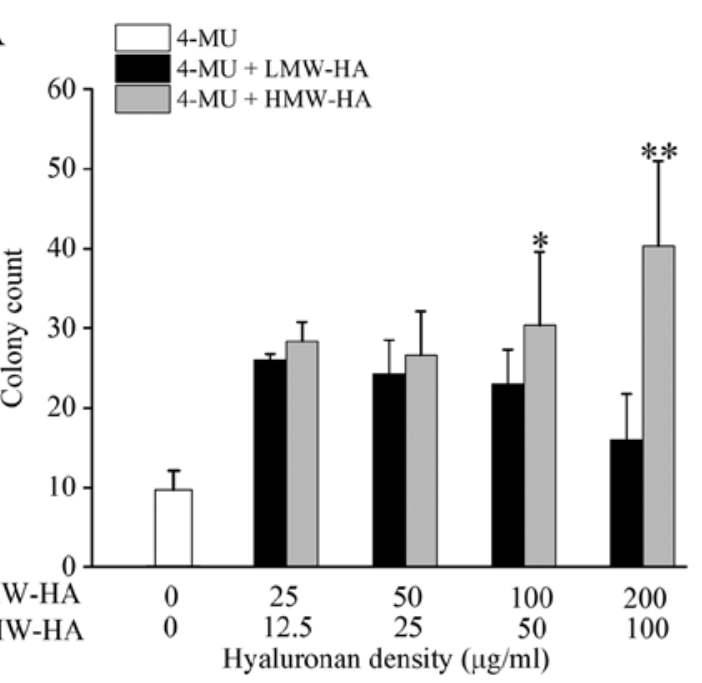

B

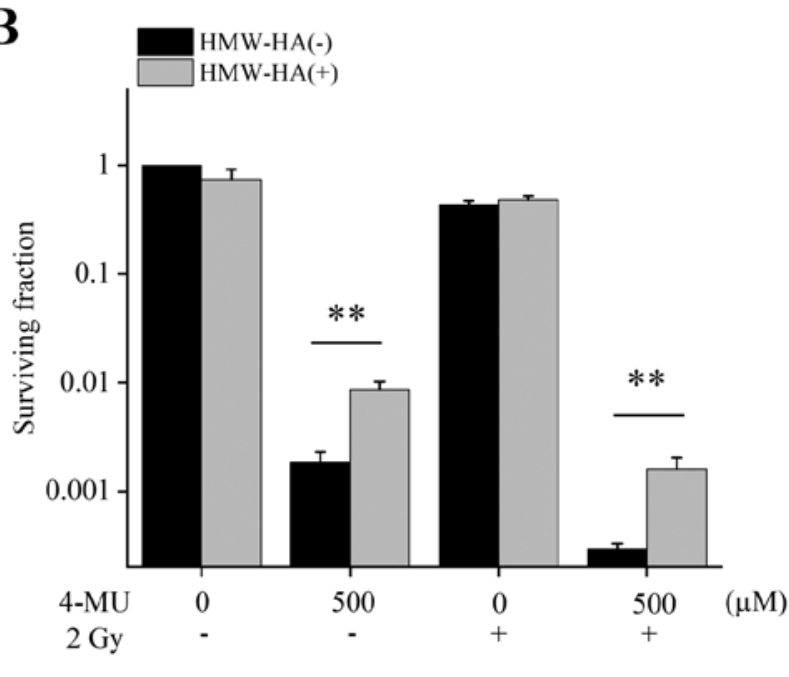

Figure 2. Radiosensitivity of 4-MU with LMW-HA or HMW-HA. (A) The number of colony counts per 20,000 cells in HT1080 cells treated with LMW-HA or HMW-HA were presented. ${ }^{*} \mathrm{P}<0.05$ and ${ }^{* *} \mathrm{P}<0.01$ vs. $500 \mu \mathrm{M} 4-\mathrm{MU}$ alone. (B) Clonogenic potency of HT1080 cells treated with HMW-HA (+) or without HMW-HA (-). Data are presented as the mean \pm standard deviation. ${ }^{*} \mathrm{P}<0.05$ and ${ }^{* *} \mathrm{P}<0.01$ vs. HMW-HA (-). 4-MU, 4-methylumbelliferone; HMW, high molecular weight LMW, low molecular weight; HA, hyaluronan.

with the combination of $500 \mu \mathrm{M} 4-\mathrm{MU}$ and 2 Gy X-ray irradiation was significantly lower than that of the cells treated with 2 Gy X-ray irradiation alone $(10.3 \pm 2.62$ vs. $19.0 \pm 3.16$ cells in the field of view) (Fig. 1E). This result suggested that invasion potential was significantly inhibited by $500 \mu \mathrm{M} 4-\mathrm{MU}$.
The effects of molecular weight of HA on surviving fraction. We evaluated the clonogenic potency of HT1080 cells exposed to 4-MU with LMW-HA or HMW-HA using the colony formation assay. The number of colony counts following administration of $500 \mu \mathrm{M} 4-\mathrm{MU}$ and exogenous HA significantly increased for both LMW-HA and HMW-HA (Fig. 2A). 


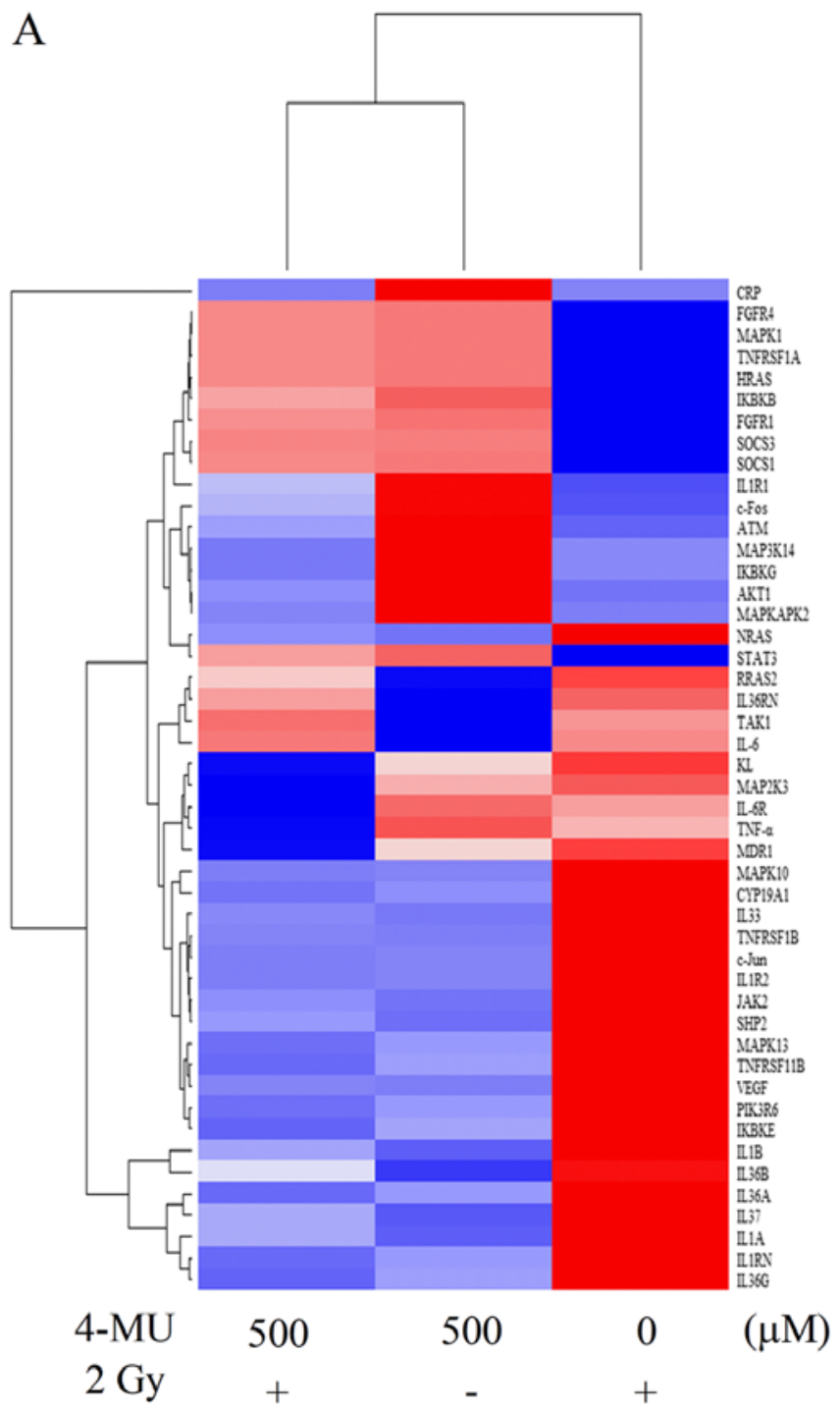

B

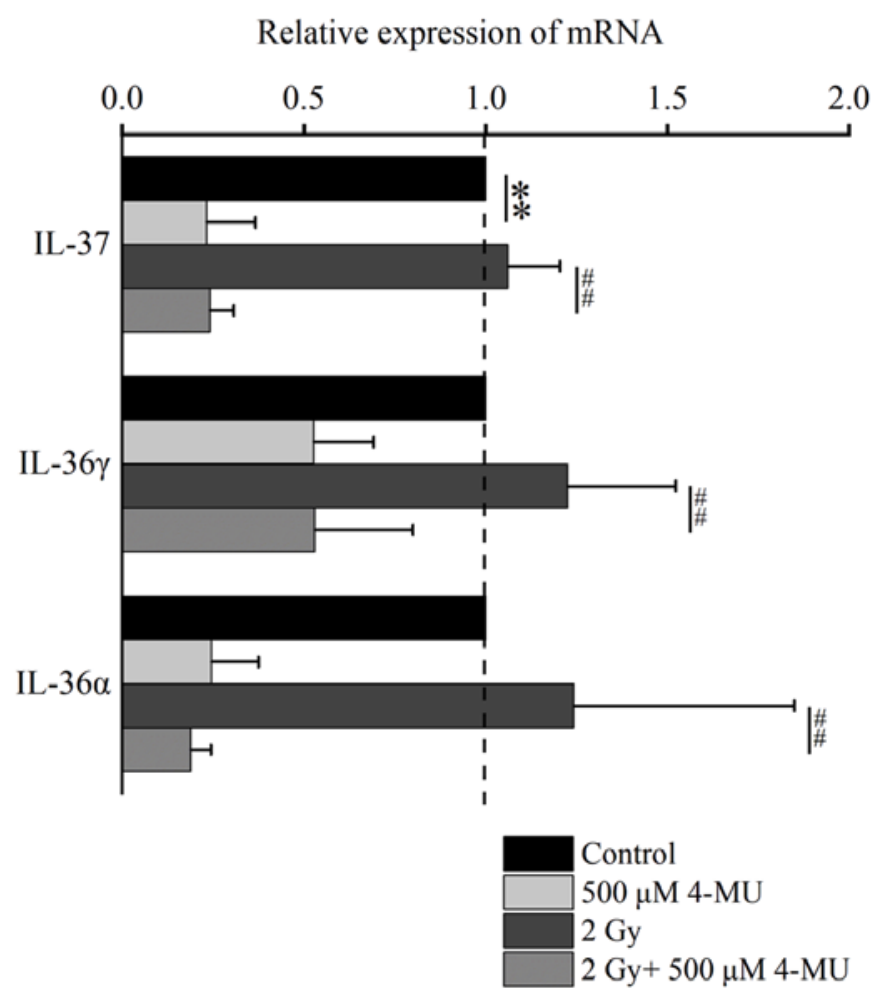

Figure 3. Cluster analysis of genes in the IL-6 signaling pathway and the mRNA expression of the identified genes. (A) Cluster analysis of 47 genes in the IL-6 signaling pathway that exhibited differential expression tendencies with $500 \mu \mathrm{M} 4-\mathrm{MU}$ and/or 2 Gy X-ray irradiation. (B) HT1080 cells treated with $500 \mu \mathrm{M}$ 4-MU and/or $2 \mathrm{~Gy}$ X-ray irradiation were cultured for $24 \mathrm{~h}$, after which the mRNA levels of IL-36 $\alpha$, IL-36 $\gamma$ and IL-37 were evaluated by reverse transcription-quantitative PCR. Red and blue squares represent fold change of $<1$ and $>1$, respectively. Data are presented as the mean \pm standard deviation. ${ }^{* *} \mathrm{P}<0.01$ vs. control and ${ }^{\# \#} \mathrm{P}<0.01$ vs. 2 Gy. IL, interleukin; 4-MU, 4-methylumbelliferone.

The number of colony counts following $25 \mu \mathrm{g} / \mathrm{ml} \mathrm{LMW}-\mathrm{HA}$ and $100 \mu \mathrm{g} / \mathrm{ml}$ HMW-HA treatments 3.1 and 4.8 folds higher than 4-MU administration alone, respectively, compared to non-HA controls. In addition, cell survival increased significantly when $100 \mu \mathrm{g} / \mathrm{ml}$ HMW-HA was administered along with 4-MU (Fig. 2B). These results suggested that exogenous HA administration suppresses the 4-MU mediated cell-killing effect, with HMW-HA being more effective than LMW-HA.

The analysis of IL-1 family mRNA following exogenous $H M W-H A$ administration. Our previous studies showed that IL-6 mRNA expression was elevated by 2 Gy X-ray irradiation, and inactivated by 4-MU (15). Clustering analysis of genes contained in the IL-6 signaling pathway revealed a total of 85 genes, with 47 genes showing differential expression pattern in each treatment. The IL-1 family genes were specifically identified in 4-MU treatment following clustering analysis of these 47 genes (Fig. 3A). Therefore, we focused on the IL-1 $\alpha,-1 \beta,-36 \alpha,-36 \gamma$, and -37 . Our previous study identified IL- $1 \alpha,-1 \beta$ and -6 , and this study newly identified IL-36 $\alpha$, $-36 \gamma$, and -37 with expression pattern like that of IL- $1 \alpha$ and $-1 \beta$. To validate the expression of the newly identified genes IL-36 $\alpha,-36 \gamma$, and -37 , we performed RT-qPCR (Fig. 3B). The mRNA expression of IL-36 $\alpha,-36 \gamma,-37$ was decreased in the presence of 4-MU.

We speculated that the genes suppressed by 4-MU were associated with HMW-HA since co-administration of exogenous HMW-HA increased the cell surviving fraction compared to non-administration HMW-HA. Therefore, we evaluated the mRNA expression level of IL-1 $\alpha,-1 \beta,-6,-36 \alpha,-36 \gamma$, and -37 by RT-qPCR following exogenous HMW-HA administration. The mRNA expression of IL-1 $\beta,-6$, and $-36 \alpha$ treated with 4-MU alone was decreased by exogenous HMW-HA administration (Fig. 4). On the other hand, the mRNA expression level of IL-1 $\alpha$, $-36 \gamma$, and -37 was significantly increased. The mRNA expression following HMW-HA co-administration in 4-MU treatment 
A $\quad$ IL-1 $\beta$

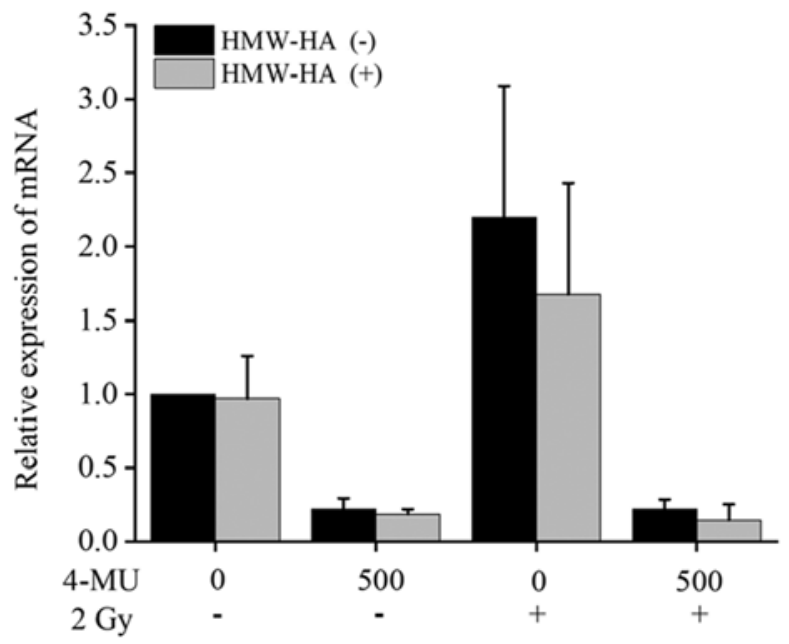

C IL-36a

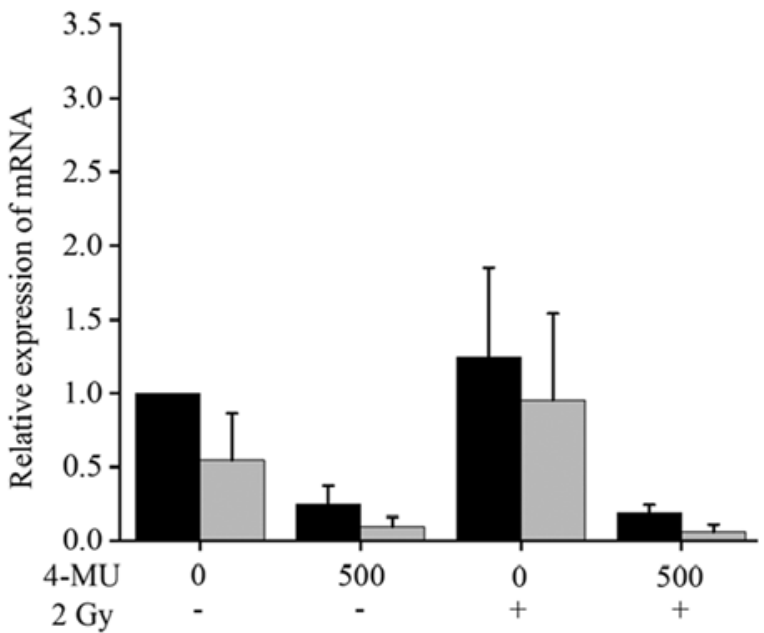

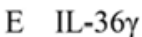

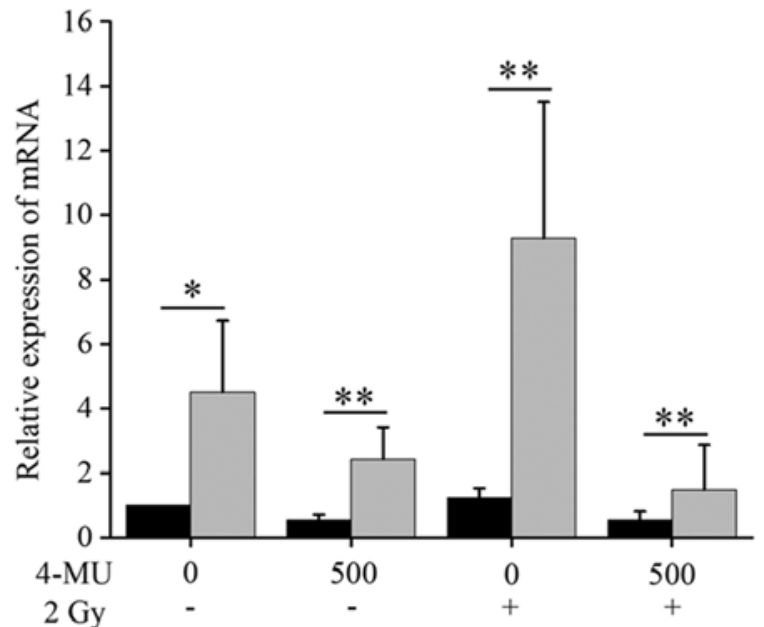

B IL-6

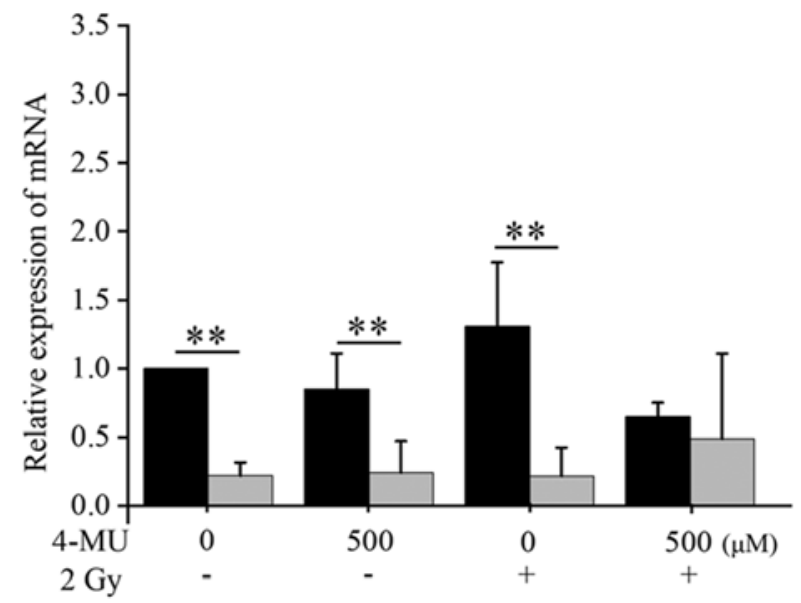

D IL-1 $\alpha$

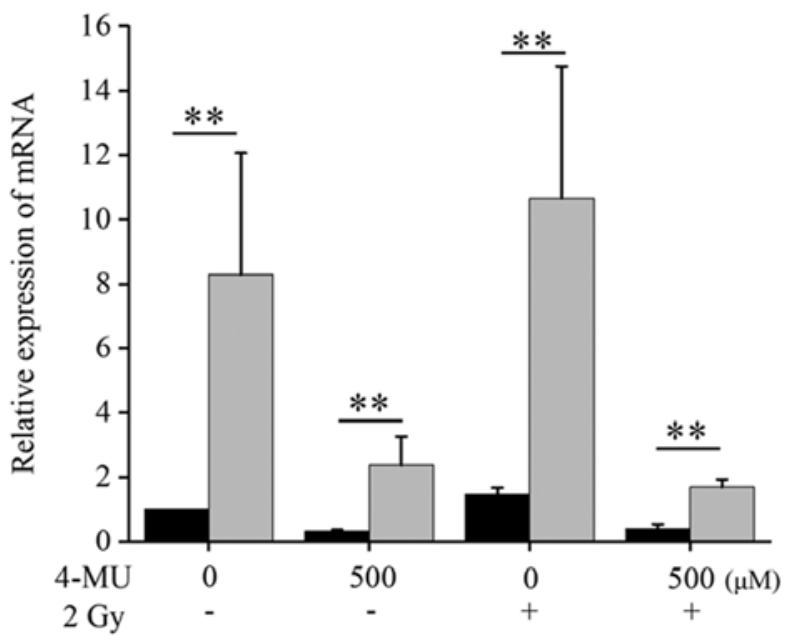

F $\quad$ IL-37

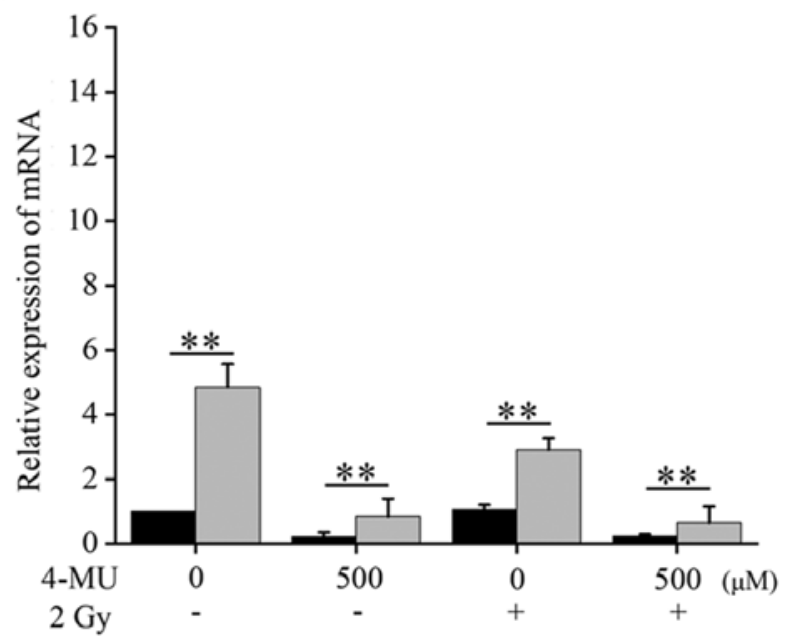

Figure 4. mRNA expression of the IL-1 family. HT1080 cells treated with $500 \mu \mathrm{M}$ 4-MU and/or 2 Gy X-ray irradiation, with or without HMW-HA, were

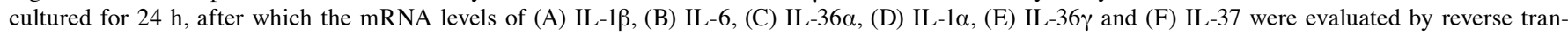
scription-quantitative PCR. Data are presented as the mean \pm standard deviation. "P $<0.05$ and ${ }^{* *} \mathrm{P}<0.01$ vs. the HMW-HA (-) group. IL, interleukin; 4-MU, 4-methylumbelliferone; HMW, high molecular weight; LMW, low molecular weight; HA, hyaluronan.

alone was increased 8-, 5- and 4-fold for IL-1 $\alpha$, IL-36 $\gamma$, and IL-37, respectively, as compared to HMW-HA non-administration. The mRNA expression following HMW-HA co-administration in the combined treatment with 4-MU and 2 Gy X-ray irradiation was also increased 4-, 3- and 2.5-fold for IL-1 $\alpha$, IL-36 $\gamma$, and IL-37, respectively, compared to HMW-HA non-administration. 
These results suggested that HMW-HA up-regulated IL-1 $\alpha$, $-36 \gamma$, and -37 expression, while 4-MU downregulated them.

\section{Discussion}

We found that 4-MU inhibits $\mathrm{HA}$ production and mRNA expression of HAS2 and -3 in HT1080 cells (Fig. 1A-C). HAS2 is involved in the synthesis and secretion of HMW-HA $(7,8)$, and it has been suggested that 4-MU could inhibit the HMW-HA production. Our results confirmed that cell-killing effect of 4-MU was suppressed by exogenously administered HMW-HA (Fig. 2A and B). In addition, we found that mRNA expression of IL-1 $\alpha,-36 \gamma$, and -37 , that are associated with IL-6 signaling, were increased by exogenous administration of HMW-HA (Fig. 4D, E and F), and that it could rescue cells from cell-killing effect of 4-MU. On the other hand, the surviving fraction following 2 Gy X-ray irradiation alone did not change with or without HMW-HA, which suggests that HMW-HA is not involved in the radio-sensitization effect of 4-MU.

The mRNA expression levels of IL-1 $\alpha,-36 \gamma$, and -37 were increased by HMW-HA. IL-1 $\alpha$ has been reported to consolidate cellular scaffolds in HT1080 cells (19). It has been reported that IL-36 has a pro-inflammatory effect in endothelial cells; while it has also been shown to have anti-inflammatory effect, its exact role remains to be determined (20). In a recent study, it was reported that IL-36 $\gamma$ activated pro-inflammatory signaling pathway on binding on the IL-36 receptor (IL-1R6), and compensated IL-1RAcp protein that is shared by IL- $1 \alpha$ and $-1 \beta(21,22)$. As a result, IL-36 $\gamma$ activated the signaling pathway like IL-1 $\alpha$ and IL-1 $\beta$. Given that IL-37 suppresses the expression of IL-1 $\beta(23,24)$, we speculated that the expression of IL-1 $\beta$ would be increased by $4-\mathrm{MU}$ because $4-\mathrm{MU}$ suppresses IL-37 expression. However, we found that IL-1 $\beta$ was also suppressed by 4-MU. Therefore, this suggested that IL-37 is not involved in the 4-MU-mediated killing effect. However, since IL-37 has not yet been fully characterized, further investigations are necessary to determine its exact role. Although anoikis, a type of programmed cell death that kills floating cells, was not measured in this study, it is possible that it was suppressed by the increased mRNA expression of IL- $1 \alpha$ and $-36 \gamma$ by exogenous HMW-HA administration. It was considered that the percentage of cells that can survive increased in spite of anoikis suppression. The results of this study suggested that HMW-HA rescues cells from the cytotoxicity of 4-MU possibly due to the enhancement of cellular scaffolds that control anoikis by IL-1 $\alpha$ and $-36 \gamma$. However, there are still many unclear points regarding the effects of the IL-36 family on the cancer cells. The IL-36 family is a factor mainly involved in skin inflammation, and our knowledge there has been no report describing its function in cancer cells. Therefore, the results of this study might provide a new facet of IL-36 cytokines. IL-6 has been shown to inhibit the oxidative stress response and DNA repair in non-small cell lung cancer cells, and promote cancer cell resistance to radioand chemotherapy (25-27). We evaluated the concentration of the reactive oxygen species (ROS) as a final messenger in bystander effects. The concentration of the intracellular ROS increased by 4-MU administration, and attenuated the effect of 4-MU by ROS scavenger dimethyl sulfoxide and c-PTIO (unpublished data). However, since IL-6 mRNA expression was suppressed by HMW-HA in this study, it was inferred that that IL-6 is not the 4-MU sensitization mechanism in HT1080 cells.

In conclusion, it was suggested that the cell-killing effect of 4-MU was inhibited by HMW-HA, while the surviving fraction with 2 Gy X-ray irradiation alone did not increase with exogenous HA. This result suggested that the radio-sensitizing effect of 4-MU and its inhibitory effect on hyaluronan synthesis are not related. Therefore, an alternate sensitivity mechanism, other than hyaluronan synthesis inhibition, possibly exists. On the other hand, this study was revealed that IL-1 $\alpha,-36 \gamma$ and -37 are involved in the cell-killing effect of 4-MU on HT1080.

\section{Acknowledgements}

Not applicable.

\section{Funding}

The present study was supported by the JSPS Grant-In-Aid for Young Scientists (grant no. 17K16413).

\section{Availability of data and materials}

The datasets used and/or analyzed during the present study are available from the corresponding author on reasonable request.

\section{Authors' contributions}

$\mathrm{KH}, \mathrm{RS}, \mathrm{KO}, \mathrm{ET}$ and $\mathrm{YH}$ conceived and designed the present study, performed the experiments and drafted the manuscript. $\mathrm{KH}, \mathrm{RS}, \mathrm{RT}, \mathrm{RF}$ and MC performed the experiments, and analyzed and interpreted the data. KO, ET, and YH critically reviewed the article. All authors read and approved the final manuscript.

\section{Ethics approval and consent to participate}

Not applicable.

\section{Patient consent for publication}

Not applicable.

\section{Competing interests}

The authors declare that they have no competing interests.

\section{References}

1. Hara T, Iwadate M, Tachibana K, Waguri S, Takenoshita S and Hamada N: Metastasis of breast cancer cells to the bone, lung, and lymph nodes promotes resistance to ionizing radiation. Strahlenther Onkol 193: 848-855, 2017.

2. Deorukhkar A and Krishnan S: Targeting inflammatory pathways for tumor radiosensitization. Biochem Pharmacol 80: 1904-1914, 2010.

3. Grivennikov SI, Greten FR and Karin M: Immunity, inflammation, and cancer. Cell 140: 883-899, 2010. 
4. Michael C. Haffner, Chiara Berlato and Wolfgang Doppler: Exploiting our knowledge of NF- $x \mathrm{~B}$ signaling for the treatment of mammary cancer. J Mammary Gland Biol Neoplasia 11: 63-73, 2006

5. Yates TJ, Lopez LE, Lokeshwar SD, Ortiz N, Kallifatidis G, Jordan A, Hoye K, Altman N and Lokeshwar VB: Dietary supplement 4-methylumbelliferone: An effective chemopreventive and therapeutic agent for prostate cancer. J Natl Cancer Inst 107: djv085, 2015.

6. Hiraga T, Ito $S$ and Nakamura H: Cancer stem-like cell marker CD44 promotes bone metastases by enhancing tumorigenicity, cell motility, and hyaluronan production. Cancer Res 73: 4112-4122, 2013.

7. Itano N, Sawai T, Yoshida M, Lenas P, Yamada Y, Imagawa M, Shinomura T, Hamaguchi M, Yoshida Y, Ohnuki Y, et al: Three isoforms of mammalian hyaluronan synthases have distinct enzymatic properties. J Biol Chem 274: 25085-25092, 1999.

8. Itano $\mathrm{N}$ and Kimata $\mathrm{K}$ : Mammalian hyaluronan synthases. IUBMB Life 54: 195-199, 2002

9. Passi A, Vigetti D, Buraschi S and Iozzo RV: Dissecting the role of hyaluronan synthases in the tumor microenvironment. FEBS J 286: 2937-2949, 2019.

10. Franklin O, Billing O, Öhlund D, Berglund A, Herdenberg C, Wang W, Hellman U and Sund M: Novel prognostic markers within the CD44-stromal ligand network in pancreatic cancer. J Pathol Clin Res 5: 130-141, 2019.

11. Li F, Hao P, Liu G, Wang W, Han R, Jiang Z and Li X: Effects of 4-methylumbelliferone and high molecular weight hyaluronic acid on the inflammation of corneal stromal cells induced by LPS. Graefes Arch Clin Exp Ophthalmol 255: 559-566, 2017.

12. Bharti R, Dey G and Mandal M: Cancer development, chemoresistance, epithelial to mesenchymal transition and stem cells: A snapshot of IL-6 mediated involvement. Cancer Lett 375: 51-61, 2016.

13. Takeda K, Fujii N, Nitta Y, Sakihara H, Nakayama K, Rikiishi $\mathrm{H}$ and Kumagai K: Murine tumor cells metastasizing selectively in the liver: Ability to produce hepatocyte-activating cytokines interleukin-1 and/or -6. Jpn J Cancer Res 82: 1299-1308, 1991.

14. Reichner JS, Mulligan JA, Palla ME, Hixson DC, Albina JE and Bland KI: Interleukin-6 production by rat hepatocellular carcinoma cells is associated with metastatic potential but not with tumorigenicity. Arch Surg 131: 360-365, 1996.

15. Saga R, Monzen S, Chiba M, Yoshino $H$, Nakamura $T$ and Hosokawa Y: Anti-tumor and anti-invasion effects of a combination of 4-methylumbelliferone and ionizing radiation in human fibrosarcoma cells. Oncol Lett 13: 410-416, 2017.

16. Saga R, Hasegawa $K$, Murata $K$, Chiba $M$ and Nakamura T: Okumura K, Tsuruga E and Hosokawa Y: Regulation of radiosensitivity by 4 -methylumbelliferone via the suppression of interleukin-1 in fibrosarcoma cell. Oncol Lett 17: 3555-3561, 2019.
17. Lokeshwar VB, Lopez LE, Munoz D, Chi A, Shirodkar SP, Lokeshwar SD, Escudero DO, Dhir N and Altman N: Antitumor activity of hyaluronic acid synthesis inhibitor 4-methylumbelliferone in prostate cancer cells. Cancer Res 70: 2613-2623, 2010.

18. Liu YY, Lee CH, Dedaj R, Zhao H, Mrabat H, Sheidlin A, Syrkina O, Huang PM, Garg HG, Hales CA, et al: High-molecular-weight hyaluronan--a possible new treatment for sepsis-induced lung injury: A preclinical study in mechanically ventilated rats. Crit Care 12: R102, 2008.

19. Nazarenko I, Marhaba R, Reich E, Voronov E, Vitacolonna M, Hildebrand D, Elter E, Rajasagi M, Apte RN and Zöller M: Tumorigenicity of IL-1alpha- and IL-1beta-deficient fibrosarcoma cells. Neoplasia 10: 549-562, 2008

20. Bridgewood C, Stacey M, Alase A, Lagos D, Graham A and Wittmann M: IL-36 $\gamma$ has proinflammatory effects on human endothelial cells. Exp Dermatol 26: 402-408, 2017.

21. Carrier Y, Ma HL, Ramon HE, Napierata L, Small C, O'Toole M, Young DA, Fouser LA, Nickerson-Nutter C, Collins M, et al: Inter-regulation of Th17 cytokines and the IL-36 cytokines in vitro and in vivo: Implications in psoriasis pathogenesis. J Invest Dermatol 131: 2428-2437, 2011.

22. Buhl AL and Wenzel J: Interleukin-36 in Infectious and Inflammatory Skin Diseases. Front Immunol 10: 1162, 2019.

23. Nold MF, Nold-Petry CA, Zepp JA, Palmer BE, Bufler P and Dinarello CA: IL-37 is a fundamental inhibitor of innate immunity. Nat Immunol 11: 1014-1022, 2010.

24. Tete S, Tripodi D, Rosati M, Conti F, Maccauro G, Saggini A, Cianchetti E, Caraffa A, Antinolfi P, Toniato E, et al: IL-37 (IL-1F7) the newest anti-inflammatory cytokine which suppresses immune responses and inflammation. Int $\mathrm{J}$ Immunopathol Pharmacol 25: 31-38, 2012.

25. Tamari $\mathrm{Y}$, Kashino $\mathrm{G}$ and Mori $\mathrm{H}$ : Acquisition of radioresistance by IL-6 treatment is caused by suppression of oxidative stress derived from mitochondria after $\gamma$-irradiation. J Radiat Res (Tokyo) 58: 412-420, 2017.

26. Chen Y, Zhang F, Tsai Y, Yang X, Yang L, Duan S, Wang X, Keng $P$ and Lee SO: IL-6 signaling promotes DNA repair and prevents apoptosis in CD133+ stem-like cells of lung cancer after radiation. Radiat Oncol 10: 227, 2015.

27. Duan S, Tsai Y, Keng P, Chen Y, Lee SO and Chen Y: IL-6 signaling contributes to cisplatin resistance in non-small cell lung cancer via the up-regulation of anti-apoptotic and DNA repair associated molecules. Oncotarget 6: 27651-27660, 2015.

This work is licensed under a Creative Commons Attribution-NonCommercial-NoDerivatives 4.0 International (CC BY-NC-ND 4.0) License. 